33/34 patients who did not return the questionnaire was determined by phone.

Results Eighty-eight of the 122 patients $(63 \pm 13$ years of age, RA duration $20.1 \pm 8.7$ years) returned the questionnaire; only $80 \%$ of whom had been seen by a rheumatologist within the last year. RA burn-out was not reported, and only 6/88 fulfilled Pinals criteria for remission. However, 26\% considered themselves 'in remission' for $8.5 \pm 6$ months, and pain was estimated at only $4.1 \pm 2.3$ on a visual analogue scale $0-10$. HAQ remained rather low $(1.11 \pm 0.84)$ for most patients; $46 \%$ had benefited from prosthetic surgery. Among the 34 patients who did not return the questionnaire, 7 had died (at a mean age of 74 years) and the other 27 had not seen a rheumatologist for a mean 3.9 years (even though only $41 \%$ considered themselves in remission).

Conclusion RA 'burn-out' was infrequent, even after 20 years of disease duration. Conversely, more than a fourth of patients considered themselves in remission, and disease activity was frequently low. These two factors probably explain why $40 \%$ of these patients had not seen a rheumatologist for at least a year. Although the HAQ level ranged from 0 to 2.6, it was often surprisingly low. Surgery could be largely responsible for the rather good functional outcome following diagnosis of erosive RA, even after more than 20 years' duration.

\section{THU0132 MORTALITY IN RHEUMATOID ARTHRITIS}

${ }^{1} \mathrm{Ml}$ Bely, ${ }^{2} \mathrm{~A}$ Apáthy. ${ }^{1}$ Pathology; ${ }^{2}$ Rheumatology, National Institute of Rheumatology, Budapest, Hungary

\subsection{6/annrheumdis-2001.1034}

Background The mortality rate in rheumatoid arthritis (RA) is higher than in the general population. Several published data exist on the prevalence of the most important complications: systemic vasculitis (SV), generalised secondary AA amyloidosis (AAa), generalised septic infection (GSI), but only a few studies discuss the causal, or additive role of these complications in the mortality of RA.

Objectives The aim of this study was to determine: (1) the proportion of basic and accompanying diseases at autopsy of RA patients, (2) the incidence of major complications, e.g. SV, AAa, and GSI, (3) the mortality due to SV, AAa, or GSI, and (4) the clinically missed major complications in RA.

Methods

Patient Population.

A randomised autopsy population of 161 in-patients (female 116, average age of 64.9 years; male 45 , average age of 66.2 years at death) with RA was studied; these patients died at the National Institute of Rheumatology between 1970 and 1992.

The basic disease, complication (s), and causes of death were determined on the basis of the autopsy findings and confirmed by a detailed review of extensive histological material (50-100 tissue blocks from each patient). The tissue samples were fixed in $8 \%$ aqueous formalin at $\mathrm{pH} 7.6$ and embedded in paraffin. Serial sections were cut and stained with haematoxylin-eosin, light green-orcein, sirius red F3BA, Ziehl-Neelsen?s method, PAS reaction, and Congo red according to Romhányi, without alcoholic differentiation, and covered by gum-arabic (1). The (AA) amyloid was determined and characterised histochemically, and by immunohistochemical reactions.

Results

- RA was the underlying cause of death in 117 cases (73\%), while in the remaining $44(27 \%)$ cases some other basic disease - such as atherosclerosis in 30 (19\%) (atherosclerosis was more or less pronounced in about $66 \%$ of the cases), tumours in $8(5 \%)$ of 18 , tuberculosis in $2(1.3 \%)$ of 13 , accidental (postoperative thromboembolism, hypertensive crisis) in $2(1.3 \%)$, acute and subacute hepatic necrosis, respectively, in $2(1.3 \%)$ cases.

- SV was found in 36 (22.4\%), AAa in 34 (21.1\%), GSI in 22 (13.7\%) of 161 RA patients. In 78 cases $(48.4 \%)$ RA was not complicated by SV, AAa, GSI, in 83 cases $(51.6 \%)$ one or more of these complications was present. In 74 patients $(46 \%)$ only one complication, and in $9(5.6 \%)$ two of the above mentioned complications existed simultaneously.

- SV led to death in $19(11.8 \%)$ of $36, \mathrm{AAa}$ in $17(10.6 \%)$ of 34, GSI in $22(13.7 \%)$ of 22 cases.

- SV was detected clinically in 7 of 36 patients (19.4 rel\%), AAa in 9 of 34 (26.5 rel\%), GSI in 10 of 22 (45.4 rel\%). Of a total of 92 complications in 83 patients only $26(28.3 \%)$ were recognised clinically.

Conclusion Major complications, e.g. SV, AAa, GSI were the main causes of death in our RA patients. They were responsible for $58(49.6 \mathrm{rel} \%)$ in 117 cases (36\% of 161 RA patients). Other RA related complications were responsible altogether for 59 (50.4\%) of 117 cases (36.6\% of 161 RA patients). The cause of death was not related directly to RA in $44(27.3 \%)$ of $161 \mathrm{RA}$ patients.

Coexisting complications modify the basic disease of RA and their own clinical manifestation, which may lead to an incorrect diagnosis or late recognition of the complications. More than half of de facto lethal complications was not diagnosed clinically.

\section{THU0133 NEW APPROACHES IN DIAGNOSIS OF SENSOR POLYNEUROPATHY IN RHEUMATOID ARTHRITIS}

BF Nemtsov, LA Smirnova, OV Simonova. Internal Medicine, Kirov State Medical Academy, Kirov, Russia

\subsection{6/annrheumdis-2001.1035}

Background Investigation of vibration perception disorders is a required method for diagnosis of sensor polyneuropathy (SPNP) in case of diabetes mellitus. SPNP is one of the most frequent extraarticular symptoms of rheumatoid arthritis (RA).

Objectives To develop and introduce a new express diagnostic method for SPNP in RA into clinical practice.

Methods 91 RA patients were studied. Of them, 91\% were females and $9 \%$ were males. The majority of patients had a severe form of RA. 49\% had extraarticular symptoms. SPNP was studied by means of measuring of vibration perception. The biothesiometer of the EG-Electronic Company was used. Lower extremities were investigated. In addition to that electroneuromyography (ENMG) was performed.

Results In more severe forms of RA the vibration perception considerably increased $(\mathrm{p}<0.01)$. In the patients with extraarticular symptoms the above disorder was more severe and more frequent as compared with the patients who had no extraarticular symptoms $(p<0.0001)$. Comparison of the two diagnostic methods showed that ENMG and biothesiometry enable to diagnose SPNP with the same frequency ( $80 \%$ and $77 \%$ relatively).

Conclusion Thus, investigation of vibration perception is as effective as the routine ENMG. This is a painless fast express method. This new method enables to diagnose extraarticular symptoms in RA very quickly. It can be used as an additional evaluation criterion of RA severity. 